A N N A L E S Annales de Bretagne et des Pays de l'Ouest

Anjou. Maine. Poitou-Charente. Touraine

110-1 | 2003

Varia

\title{
Les congrégations jésuites du collège Saint-
} Thomas

Contribution à l'histoire religieuse de Rennes sous l'Ancien Régime

\section{Matthieu Heim}

\section{OpenEdition}

\section{Journals}

Édition électronique

URL : http://journals.openedition.org/abpo/1469

DOI : $10.4000 /$ abpo.1469

ISBN : 978-2-7535-1489-8

ISSN : 2108-6443

\section{Éditeur}

Presses universitaires de Rennes

\section{Édition imprimée}

Date de publication : 20 mars 2003

Pagination : 79-92

ISBN : 978-2-86847-811-5

ISSN : 0399-0826

Référence électronique

Matthieu Heim, "Les congrégations jésuites du collège Saint-Thomas », Annales de Bretagne et des Pays de l'Ouest [En ligne], 110-1 | 2003, mis en ligne le 20 mars 2005, consulté le 01 mai 2019. URL http://journals.openedition.org/abpo/1469; DOI : 10.4000/abpo.1469 


\title{
Les congrégations jésuites du collège Saint-Thomas
}

\section{Contribution à l'histoire religieuse de Rennes sous l'Ancien Régime ${ }^{1}$}

\author{
Matthieu HEIM \\ Professeur de lycée à Vitry-sur-Seine
}

Si beaucoup de Rennais ignorent que les Jésuites ont vécu dans leur ville sous l'Ancien Régime, bien moins nombreux encore sont ceux qui savent que le collège Saint-Thomas abrita jadis des congrégations mariales. À leur décharge, on peut rappeler que les missions en milieu rural d'un Le Nobletz ou d'un Maunoir ont eu tendance à éclipser les congrégations urbaines, moins spectaculaires, et que, s'agissant des Jésuites, l'opinion de B. Pocquet selon laquelle " [leurs] collèges n'ont pas d'histoire ${ }^{2}$ " a longtemps prévalu chez les historiens. C'est donc dans la perspective choisie par les Jésuites eux-mêmes qu'il convient de replacer le collège Saint-Thomas : celle d'un centre d'enseignement bien sûr, mais aussi et surtout d'apostolat ${ }^{3}$.

Saint-Thomas, où s'établissent les Jésuites en 1604, était la première installation de l'ordre en Bretagne ${ }^{4}$. Avec Louis-le-Grand et les collèges de La Flèche et Rouen, il faisait partie des quatre plus grands collèges de la province de Paris ${ }^{5}$. Mais c'était avant tout un foyer missionnaire. Son empla-

1. Le présent article est tiré d'un mémoire de maîtrise intitulé Ad Majorem Virginis Gloriam. Les congrégations mariales du collège des Jésuites de Rennes (1662-1762) et soutenu en 1997 sous la direction d'Alain Croix à l'Université de Rennes 2.

2. PoCquet, Barthélémy, Histoire de Bretagne, Rennes, 1913, chap. 36, p. 585-586.

3. L'arrivée des Jésuites dans une ville au début du XvII ${ }^{\mathrm{e}}$ siècle, après les troubles des guerres de religion, a souvent été interprétée comme un signe de reconquête catholique. D'après Louis Châtellier, les congrégations jésuites, toutes dédiées à la Vierge, procèdent clairement du militantisme anti-protestant (L'Europe des dévots, Paris, 1987, p. 22).

4. DaINVILLE, François de, "Projet d'établissement des Jésuites en Bretagne sous Louis XIII ", Mémoires de la Société d'Histoire et d'Archéologie de Bretagne, t. 195, p. 37-47 : « Ils se portent de préférence vers les villes de magistrature, Parlements et présidiaux. Dans ce milieu, ils rencontrent sinon le gros des effectifs, du moins les meilleures têtes de leurs classes."

5. Michel, Joseph, Claude-François Poullart des Places, fondateur de la Congrégation du Saint-Esprit (1679-1709), Paris, 1902, p. 17 : « Rennes possédait un établissement d'ensei- 
cement le rappelle encore à qui veut bien le voir. Situé au sud d'une Vilaine fantasque, il obligeait en effet les fils de bonne famille, toutes concentrées alors entre la cathédrale et le Parlement, à se rendre dans les rues populaires et populeuses de la ville basse ${ }^{6}$. D'autre part, sa chapelle aux dimensions impressionnantes ${ }^{7}$ se posait sur un axe nord-sud comme la rivale symétrique de l'église Saint-Germain et, sur un axe est-ouest, comme un contrepoids symbolique à la vieille cathédrale qui menaçait ruine depuis le $\mathrm{XVI}^{\mathrm{e}}$ siècle $^{8}$. Quasiment équidistante du nord et du sud de la ville - les remparts occupaient au nord l'actuelle rue des Fossés et au sud l'actuel boulevard de la Liberté - elle réunissait à la manière d'un trait d'union les riches quartiers du centre et les faubourgs agrestes moins fortunés ${ }^{9}$. Un peu comme si ses transepts eussent été les bras d'un Christ en croix généreusement ouverts pour rédimer toute la cité. La stratégie missionnaire des bons pères se lisait donc dans la géographie même de la ville. À l'inverse des confréries traditionnelles dont le champ d'action ne dépassait guère la vieille enceinte, le positionnement des congrégations mariales était d'emblée plus universel.

\section{Les congréganistes de Saint-Thomas}

Saint-Thomas abritait en effet, outre les congrégations d'écoliers communes à tous les collèges de la Compagnie de Jésus, deux congrégations dédiées à la Vierge : celle des messieurs et celle des marchands et artisans. Ces deux sodalités s'adressaient clairement à l'élite de la ville puisqu'elles " visaient à ramener les classes dirigeantes et aisées, bourgeoisie et noblesse, à la foi catholique, avec l'espoir d'infuser par leur intermédiaire la vie chrétienne dans tous les rouages de la société ${ }^{10}$ ". À cet égard, la comparaison de l'ordre d'institution des congrégations à Rennes et à Nantes permet de prendre conscience du poids de la robe dans la capitale bre-

gnement dont la renommée s'étendait bien au-delà des frontières de la province. Tant par le nombre de ses élèves - tout près de 3000 - que par la valeur de ses professeurs, le Collège Saint-Thomas tenait une place de choix parmi tous ceux que les Pères de la Compagnie de Jésus dirigeaient dans le royaume. " Parmi les professeurs, citons par exemple le R. P. J. H. Premare qui partit pour la Chine en 1698 et fut l'initiateur des études sinologiques, ou bien encore le R. P. Charles Porée qui y enseigna les humanités et la rhétorique avant de devenir le maître de Voltaire à Louis-le-Grand.

6. Certains, à tort, s'en sont étonnés. Cf. MEYER, Jean (dir.), Histoire de Rennes, Privat, p. 256 : "symbole inattendu, et ce n'est pas le moindre des paradoxes rennais, le collège des Jésuites se situe au beau milieu de la zone de criminalité. "

7. Il s'agit de l'actuelle église de Toussaints.

8. Pour Louis Châtellier, dans L'Europe des dévots, p. 67-68, « le sanctuaire élevé par la Société de Jésus prend les caractères de seconde grande église destinée à relayer, en direction des faubourgs et des alentours, l'action quelquefois un peu alanguie de la cathédrale, de la collégiale ou de l'église paroissiale. "

9. Pour un tableau détaillé de Rennes et de ses faubourgs à cette époque, voir le livre de Philippe Jarnoux, Les bourgeois et la terre, Rennes, PUR, 1996, p. 320.

10. PoCQuet, B., " Histoire religieuse de Rennes aux XVI ${ }^{\mathrm{e}}$ et XVII ${ }^{\mathrm{e}}$ siècles ", Mémoires de la Société d'Histoire et d'Archéologie de Bretagne, 1971. 
tonne. Alors que la congrégation des artisans précède de six ans celle des messieurs à la résidence de Nantes ${ }^{11}$, à Rennes la congrégation des messieurs précède celle des artisans de quarante-trois ans! La première est établie officiellement à 1619, la seconde en 1662.

Pour être admis dans l'une comme dans l'autre congrégation, le candidat doit d'abord être présenté par un congréganiste qui se porte garant de sa moralité, puis subir une période probatoire de durée variable qui se conclut, en principe, par une consécration à la Vierge qu'on appelle tantôt " protestation " chez les messieurs tantôt " bon propoz " chez les marchands ${ }^{12}$. Chez ces derniers, en outre, le futur congréganiste doit être impérativement marié et maître de sa vacation. Après quoi l'on est membre de la congrégation jusqu'à sa mort, à moins de faire l'objet d'une mesure d'exclusion - celle-ci étant le revers scandaleux de celle-là. L'accompagnement du mourant et l'organisation d'un somptueux cortège funèbre figurent en effet parmi les objectifs premiers de toute sodalité. Mais ces faveurs se méritent : l'assiduité aux assemblées doit être prouvée et les cotisations à jour. En creux se révèlent ici les deux motifs principaux d'exclusion, à savoir l'absentéisme et les dettes. S'y ajoute pour les marchands toute infraction à l'un des trois articles suivants du règlement :

"Le Premier est d'avoir eu part à quelque Complot, Sédition, Motion ou Rebellion populaire contre l'authorité du Roy et des Magistrats. Le second, d'avoir posé un Exploit ou pledé [plaidé] contre un Confrère, quelque droit qu'on peut y avoir, sans avoir treté [traité] de l'affaire dont il est question avec le R. P. Directeur, ou le Préfet de la Congrégation pour chercher les moyens de s'accorder par voyes aimables. Le troisième, d'avoir esté en Masque et déguisé comme l'on fait à Carnaval ${ }^{13}$."

Quelquefois, enfin, des congréganistes sont chassés « pour leurs yvrogneries publiques et scandaleuses ${ }^{14}$ ". Pour assurer la discipline du groupe, la congrégation est donc structurée selon une rigoureuse hiérarchie. Aux côtés d'un bon père qui en assure la direction spirituelle, on trouve à la tête du groupe un préfet secondé par des officiers et un conseil. L'accès à ces

11. Canal, Séverin, "La Compagnie de Jésus au diocèse de Nantes ", Mémoires de la Société d'Histoire et d'Archéologie de Bretagne, 1945, p. 83-195 et 1946, p. 91-158.

12. Cette promesse n'a pas la force d'un vœu et doit donc être renouvelée chaque année. La formule, en latin, est la suivante : "Sancta Maria, Mater Dei et Virgo, Ego N.N. te hodie in Dominam, Patronam et Advocatam eligo. Firmiterque Statuo ac Propono me numquam te derelicturum; neque contra te aliquid unquam dicturum aut facturum, neque permissurum ut a meis subditis aliquid contra tuum honorem umquam agatur. Suscipe me igitur in servum perpetuum, adsis mihi in omnibus actionibus meis, nec deseras in hora mortis. Amen. " La traduction française de l'époque donne ceci : "Sainte Marie, Mère de Dieu et Vierge, Je N.N. vous choisis aujourd'huy pour ma Maîtresse, pour ma Patrone et pour mon Avocate. Et je fais une ferme résolution de ne vous abandonner jamais, de ne jamais rien dire ou faire de contraire à vos intérests, et de ne jamais permettre qu'aucun de ceux qui me sont, ou seront soumis, intéresse votre honneur. Agréez-moy donc à jamais pour votre serviteur. Assistez-moy dans toutes mes actions et ne m'abandonnez point à l'heure de la mort. Ainsi soit-il. "

13. Arch. dép. d'Ille-et-Vilaine, 3D-15, Registre des élections.

14. Idem, Cahier des délibérations, 27 janvier 1686. 
postes diffère d'une congrégation à l'autre. Chaque année, à la veille de la Purification - puisqu'elle est placée sous le vocable de cette fête - l'assemblée des messieurs départage les quelques personnes que le préfet sortant et ses deux assistants ont retenues de concert avec le R. P. directeur pour leur succéder. Puis, à partir de 1708, ces postes font l'objet, comme tous les autres, d'une nomination par le conseil. C'est donc bien dans son ancienne acception, celle de cooptation, que le terme " élection " doit être compris dans la première congrégation. Les marchands, eux, mettent empiriquement en place, dès les premières années $d u$ XVIII ${ }^{\mathrm{e}}$ siècle, un système de promotion interne définissant une manière de cursus honorum. Ceux qui parviennent ainsi à la préfecture, après être passés par tous les postes subalternes, ont pour eux l'avantage de connaître tous les rouages de la sodalité. Le principe de la cooptation, appliqué à l'extérieur comme à l'intérieur de la congrégation, explique sans nul doute la relative stabilité du nombre des confrères ainsi que leur remarquable homogénéité sociale.

Par-delà la distorsion permanente entre les nombres théorique et effectif des congréganistes, il ressort des sources que la congrégation des marchands et artisans fut toujours plus nombreuse que celle des messieurs. Comptant plus d'une centaine de membres dès l'origine, sa population augmente $\mathrm{au} \mathrm{XVIII}{ }^{\mathrm{e}}$ siècle - si toutefois l'accroissement de ses instances dirigeantes est bien la traduction d'un accroissement de sa base (37 officiers dans les années 1660 contre 48 en moyenne dans les années 1730). La congrégation des messieurs, si l'on en croit ses Annales, a pu, elle aussi, compter plus de cent membres à ses débuts. Toutefois, dès le début des années 1670, l'affluence réelle ne dépasse plus jamais la centaine de personnes ${ }^{15}$. Elle enregistre par ailleurs une brusque chute de ses effectifs en 1676, année qui fait suite à l'exil du Parlement à Vannes, puis un pic en 1691, soit juste après le retour de celui-ci à Rennes. La coïncidence entre les vicissitudes du Parlement de Bretagne et la vie de la congrégation met en évidence la grande homogénéité sociale de cette sodalitas civium aut nobilium - congrégation de bourgeois ou de nobles. Le primat des magistrats, du Parlement et du Présidial, dans la société rennaise de l'époque se trouve vérifié à l'échelle de la congrégation : c'est à eux que revient le plus souvent l'honneur d'être préfet. Ils ne représentent pourtant, à la fin du XVII siècle, que 13,68 \% des confrères. Les avocats et les procureurs sont alors les plus nombreux (33,24 \%). Si l'on ajoute aux magistrats et aux avocats les greffiers, les huissiers et les notaires, le monde de la justice totalise une bonne moitié des membres $(54,46 \%)^{16}$. Viennent ensuite, par ordre décroissant : le haut négoce et l'artisanat de luxe (18,71\%), le clergé (2,79\%) et les professions médicales (2,23\%). L'état civil des autres membres est soit trop éclaté pour être significatif $(1,95 \%)$ soit inconnu $(19,83 \%){ }^{17}$. À la sur-

15. Les cotisations aux grandes fêtes d'obligation que sont la Purification et l'Assomption le montrent clairement.

16. La mainmise de la robe apparaît plus nette encore lorsqu'on sait que nombre de membres enregistrés comme marchands cumulent en réalité cette activité avec la profession d'avocat. 
représentation des robins chez les messieurs fait écho celle des petits patrons chez les artisans. "Maîtres de leur vacation ", ils jouissent d'une réputation et d'une clientèle suffisantes pour faire preuve d'une honnête aisance. (Certains de ces notables se retrouveront dans les généraux des paroisses de Saint-Germain et Toussaints après la dissolution de la congrégation). Aucun ne peut cependant rivaliser avec l'aristocratie de l'artisanat parvenue à pénétrer la congrégation des messieurs, à l'instar de certains orfèvres, du libraire Vatar ou encore du cirier Nicolazzo fournissant moult couvents dont la ville regorge à l'époque.

Les deux confréries révèlent d'importantes différences. La première est sociale : les messieurs se recrutent dans les trois ordres tandis que les marchands proviennent exclusivement du tiers état. La seconde est sexuelle : alors que les marchands accueillent bientôt des femmes, la congrégation des messieurs, où la présence cléricale est plus forte, demeure exclusivement masculine. La dernière différence est culturelle : si l'usage du latin a tendance à se perdre chez les messieurs ${ }^{18}$, c'est l'alphabétisation elle-même qui pose problème chez les marchands. À preuve, la formule de consécration à la Vierge est lue à haute voix à chaque assemblée " de sorte que ceux qui ne sçavent pas lire la puissent apprendre par cœur ${ }^{19}$ ". Les deux sodalités se ressemblent néanmoins, ainsi que le révèlent leurs trésoreries, en ceci qu'elles réunissent chacune l'élite la plus fortunée des publics qu'elles ciblent.

\section{Le fonctionnement de la congrégation}

Autant la trésorerie des marchands fonctionne comme celle d'une confrérie ordinaire, autant celle des messieurs s'en éloigne fortement. Chez les messieurs, les recettes constituées par les cotisations recueillies à la Purification ${ }^{20}$ et à l'Assomption ${ }^{21}$ s'avèrent plutôt médiocres. Elles contrebalancent difficilement les dépenses engagées pour l'entretien d'une congrégation aussi prestigieuse. Ainsi, rien qu'entre 1663 et 1718, la congrégation doit-elle s'endetter quatorze fois, soit une fois tous les quatre ans en moyenne. En fait, seuls les dons en argent ou en nature (chandeliers, chasubles, tapisseries...), en permettant d'économiser sur le fond de roulement, apportent la souplesse financière nécessaire pour faire face aux imprévus (réfection de la toiture, remplacement des serrures et des vitres cassées, etc.) Chez les marchands, les quêtes effectuées aux assemblées hebdomadaires représentent la quasi totalité des recettes; les frais occasionnés par la fête annuelle de la Nativité, les principales dépenses. L'ensemble des sommes manipulées excède rarement la centaine de livres.

17. Les sources, lacunaires, n'indiquent parfois qu'un nom sans autre précision.

18. À partir de 1705, l'acte de consécration peut se faire en français.

19. Arch. dép. d'Ille-et-Vilaine, 3D-15 et 16, Cahier des délibérations, $\mathrm{f}^{\circ} 6 \mathrm{r}^{\circ}, 23$ décembre 1668 .

20. C'est la fête annuelle de la congrégation des messieurs.

21. C'est la fête principale de la Vierge. 
L'analyse des recettes, en confirmant la stabilité relative des effectifs, imprime toutefois une nette différence entre les deux congrégations jésuites et les confréries traditionnelles qui « connaissent un fort déclin à partir des années 1720-173022".

Le patrimoine des deux congrégations est renfermé dans deux chapelles distinctes ${ }^{23}$. Un incendie, le 8 février 1712, a détruit celle des marchands ainsi que la bibliothèque du collège qui la surmontait. Cet événement fournit une indication assez précise quant à la fortune de cette sodalité puisque les marchands évaluèrent alors les pertes à " plus de vingt mil Livres tant en argenterie, ornements que Meubles ${ }^{24}$ ". Obligés pour un temps de tenir leurs assemblées dans une salle du collège, les marchands placent, en 1716, 3000 livres sauvées des flammes chez le plus grand banquier rennais d'alors, Michau de Montaran. Cette somme sert en 1718 à financer en partie la construction d'une nouvelle chapelle qui sera utilisée dès la Toussaints 1719. La chapelle des messieurs aurait pu brûler tout aussi bien dans la mesure où, comme l'indique un inventaire de 1713, elle était entièrement parquetée et, depuis 1710, lambrissée jusqu'à la voûte " en forme d'anse de panier ${ }^{25}$ ". Construite en 1655, elle avait été décorée aux frais des confrères. Le célèbre rétablier lavallois François II Houdault réalisa le maître-autel en $1660^{26}$ et son associé habituel, le sculpteur angevin La Barre, les statues. Le plafond fut peint en 1715 par l'Italien

22. MinoIs, Georges, "Le réseau des confréries pieuses est-il un indice valable du sentiment religieux?", Annales de Bretagne et des Pays de l'Ouest, t. 90, 1983. Voir aussi PoulPIQuET, Dominique de, "Le couvent des Grand Carmes à Rennes aux XVII et XVIII ${ }^{\mathrm{e}}$ siècles ", Mémoires de la Société d'Histoire et d'Archéologie de Bretagne, 1986, t. 63, p. 234. À titre de comparaison, chez les Carmes qui, au XVIII ${ }^{\mathrm{e}}$ siècle, " $\mathbf{s}$ 'affichent parmi les plus grosses fortunes religieuses de Rennes ", les cinq confréries (dont une dédiée à la Vierge) sont généralement déficitaires.

23. À l'inverse des messieurs qui possèdent leur propre chapelle, les marchands doivent partager la leur, tout au moins au début, avec la congrégation des écoliers. L'examen du plan de Rennes établi par Forestier en 1726 montre que ces deux chapelles devaient posséder des dimensions remarquables car, en général, les chapelles des congrégations jésuites excédaient rarement 20 mètres de long. Voir Morsy, Pierre, Les églises des Jésuites de l'ancienne assistance de France, Rome, 1958, p. 312.

24. Arch. dép. d'Ille-et-Vilaine, 3D-15, Registre des élections, p. 36. Certains congréganistes ont vu dans cet incendie une punition divine puisqu'ils demandèrent au père provincial De La Granville, lors de sa visite à Rennes en 1719, qu'on n'y représente plus aucune pièce de théâtre, " ce quy par le passé avoit attiré la malédiction de Dieu sur elle " (Cahier des délibérations, $\mathrm{f}^{\circ} 47 \mathrm{v}^{\circ}, 6$ septembre 1719).

25. Arch. dép. d'Ille-et-Vilaine, 3D-14, Inventaire des choses appartenantes à la Congrégation des Messieurs.

26. Arch. dép. d'Ille-et-Vilaine, 3D-14, délibération du $1^{\text {er }}$ juillet 1659 et E-415, minute Bertelot du 2 juillet 1659. Exécuté pour 2600 livres, ce retable est aujourd'hui disparu. Toutefois, les deux autels conservés actuellement dans le transept de l'église de Toussaints et réalisés par le même rétablier en 1672 et 1674 pour la somme totale de 4000 livres peuvent en donner une idée approchante. Pour plus de détails, voir : SALBERT, Jacques, Les ateliers de rétabliers lavallois aux XVII et XVII ${ }^{e}$ siècles, Paris, 1976, p. 245-246; PELLETIER, Yannick, Les retables, Rennes, 1984, p. 133; MALOUBIER-TournIER, P., " Les retables du XVII e et $\mathrm{XVIII}^{\mathrm{e}}$ siècle en Ille-et-Vilaine ", Annales de Bretagne et des Pays de l'Ouest, t. 69, 1962. 
G. B. Gherardini ${ }^{27}$. L'esthétique de l'ensemble était, sans aucun doute, d'inspiration baroque. En effet, aux côtés du style rocaille - présent avec un reliquaire " en forme de grotte de coquillages " - l'esprit des "vanités " domine : huit tableaux richement encadrés évoquent les martyrs jésuites au Canada, Jésus au jardin des oliviers ou saint François-Xavier agonisant; les chandeliers d'ébène vernissé sont incrustés de " testes de mort et ossements blancs "..., etc. À l'évidence, les messieurs appréciaient l'association étroite, mais riche de sens, des marques du faste et des signes mortifères.

Cette richesse mobilière, remarquons-le, ne peut que laisser perplexe devant le produit de la vente des biens du collège et des congrégations saisis en $1762: 29534$ livres 4 sols et 6 deniers. Sachant, en effet, que l'argenterie représente à elle seule 21957 livres 13 sols et 6 deniers ${ }^{28}$ et que la prisée effectuée chez les messieurs en vue de la liquidation recense seulement 12 tableaux $^{29}$ (contre 26 dans l'inventaire de 1713!), il n'est pas interdit de penser que les congréganistes ont pu soustraire une partie de leurs biens à la mise sous séquestre ${ }^{30}$. En agissant de la sorte, ils n'auront fait que suivre l'exemple de leurs homologues de la résidence de Nantes qui ont tantôt vendu tantôt remis à des personnes de confiance un certain nombre d'effets et de meubles ${ }^{31}$. Selon toute vraisemblance, les congréganistes ont donc attendu d'être réduits à la dernière extrémité pour envisager l'idée de leur suppression tant celle-ci leur semblait grotesque; au contraire, ils pensaient revenir bientôt pour augmenter la gloire de Dieu et continuer de répandre leurs bienfaits.

Les messieurs étendaient leur action charitable aux pauvres, aux malades et aux prisonniers. S'agissant des pauvres, cette assistance prenait trois formes principales. D'abord, le rite du lavement des pieds le jeudi saint offrait chaque année aux congréganistes l'occasion d'imiter le Christ. En ce " Jeudy Absolu ", ainsi qu'ils l'appelaient, les messieurs conviaient à déjeuner et servaient eux-mêmes à table douze pauvres vieillards après leur avoir lavé les pieds. Ensuite, deux fois par an - à l'Assomption et à la Purification - ils apportaient un secours financier à des " pauvres honteux ". Cette

27. BERGOT, François, L'église de Toussaints de Rennes, Rennes, 1973. Nous devons cette information à Monsieur Xavier Ferrieu qui la reprend dans son Histoire de Rennes, Éditions Jean-Paul Gisserot, 2001, p. 47.

28. Arch. dép. d'Ille-et-Vilaine, 3D-9. L'argenterie fut intégralement acquise par le comte de La Garlaye - l'évêque de Rennes, Desnos, lui servant de prête-nom - et déposée au Grand Séminaire afin d'être restituée aux congrégations dans l'hypothèse où elles seraient rétablies sous quarante ans, " quand bien même les Jésuites ne le seroient pas ". Elle fut finalement vendue en 1770 à l'orfèvre Rahier.

29. Archives municipales de Rennes, liasse 287 , Titres concernant le collège de la ville de Rennes (1650-1762).

30 Si les preuves formelles font défaut, les suspicions des huissiers allaient déjà dans ce sens, ainsi que le montre le procès-verbal du 10 mai 1762. Cf. DURTELLE-DE-SAINT-SAUVEUR, Geneviève, loc. cit., p. 218 (note infrapaginale).

31. Canal, Séverin, "La Compagnie de Jésus au diocèse de Nantes", Mémoires de la Société d'Histoire et d'Archéologie de Bretagne, 1945, p. 148. 
expression désignant « les gens de famille qui souffrent nécessité sans oser le découvrir " (P. Hardouin), cette aide, si on la voulait efficace, devait être précédée d'une enquête des congréganistes quartier par quartier. Enfin, la Marmite des Pauvres manifestait indirectement, mais plus durablement, la charité des messieurs. En apparence, rien ne rattache cette œuvre laïque fondée en 1643 par quelques femmes de la haute société rennaise aux assemblées Jésuites $^{32}$. Mais ses statuts - rédigés en 1674 seulement, après que saint Vincent de Paul fut venu à Rennes mettre à sa disposition les Filles de la Charité ${ }^{33}$ - montrent que les douze premiers administrateurs étaient des chevaliers de la Vierge ${ }^{34}$. Mieux : afin de loger les sœurs de la charité, la Marmite fit l'acquisition le 12 août 1683 d'une maison à l'angle de la rue du Griffon et de la rue des Lauriers auprès du congréganiste Jean-François Ravenel du Plessis. Ce dernier profita de cette vente pour effectuer un don important puisque 2500 livres sur les 4800 du montant officiel de la vente revinrent à la Marmite. En 1694, enfin, les directeurs de la Marmite, tous congréganistes, confièrent les 4050 livres de dons récoltés en vue de favoriser l'installation de deux sœurs grises envoyées de Paris en renfort au seigneur de La Falluère, congréganiste lui aussi, en contrepartie de 225 livres de rente annuelle. Les chevaliers de la vierge, on le voit, occupaient discrètement mais sûrement le cœur du dispositif d'assistance aux pauvres ${ }^{35}$.

Leur charité ne leur faisait pas oublier cependant les malades et les prisonniers. Si la congrégation des marchands a ses habitudes à l'hôpital SaintYves, celle des messieurs préfère l'hôpital général. Là encore il semble que les messieurs aient fait valoir leur influence. On sait que c'est parmi les congréganistes jésuites que les hôpitaux généraux trouvaient le plus souvent leurs premiers bienfaiteurs et administrateurs. Rennes n'échappe pas à la règle : l'économe de l'hôpital général, Monsieur de Pontgallou, cotise chez les messieurs de 1682 à 1699. Les nourritures apportées aux malades étaient à la fois terrestres et spirituelles. Le motif principal des visites était, en effet, de convertir les pécheurs et de préserver ceux dont l'âme était jugée en danger. Aux moribonds on apprenait à mourir [sic], aux autres on donnait des leçons de catéchisme et des conseils sur la manière de se confesser et de communier. Les prisonniers, quant à eux, étaient incités à mener une vie quasi-monacale et à offrir leurs journées à Dieu pour racheter leurs fautes ${ }^{36}$. Sur ce chapitre, l'espacement progressif des visites est, nous semble-t-il, le

32. Hardouin (P.), "La Marmite des Pauvres ", Mémoires de la Société Historique et Archéologique d'Ille-et-Vilaine, t. 70, 1956.

33. Pocquet du Haut-Jussé (B.), "Vincent de Paul à Rennes et les Filles de la Charité, rue du Griffon ", Mémoires de la Société Historique et Archéologique d'Ille-et-Vilaine, t. 74, 1964.

34. Arch. municipales de Rennes, 1-Q 18, Notice sur les administrateurs de la Marmite des Pauvres.

35. La marmite des Pauvres, qui connut plusieurs avatars (Bureau de bienfaisance sous la Révolution, Bureau d'aide sociale à partir de 1956) est l'une des rares institutions à avoir transmis jusqu'à nos jours un peu de l'" esprit " des congrégations mariales.

36. Les deux prisons de Rennes étaient alors La Feuillée (encore appelée prison SaintMichel et toujours visible aujourd'hui au fond de l'impasse Rallier-du-Baty) et la Tour-leBât, vestige des fortifications qui se trouvaient à l'emplacement de l'actuelle rue des Fossés. 
fait majeur à relever. Tandis qu'en 1659 les messieurs délèguent quatre membres pour visiter chaque semaine les hôpitaux et les prisons, en 1712 la visite des prisons n'a plus lieu qu'une fois tous les trois mois en moyenne. Force est d'ajouter néanmoins que l'assistance en milieu carcéral n'est pas délaissée pour autant par les confrères : elle prend seulement la forme nouvelle d'une intervention en vue de faire libérer les prisonniers pour dettes. Concrètement, la congrégation demande au créancier lésé, promesse de paiement à l'appui, de renoncer à exercer la contrainte par corps ou bien d'autoriser, le cas échéant, l'élargissement du prisonnier. Le trésorier, cela va de soi, tient dans ces démarches le premier rôle. Ce n'est donc pas un hasard si sont désignés à ce poste des juristes aguerris, à l'instar de l'avocat René-Raoul Drouin qui en 1759 se déclare "receveur de la Congrégation des Messieurs établie chez les Jésuites " et " professeur dans les facultés de cette ville ".

Attention toutefois à ne pas se tromper : la charité des confrères est toujours sélective et intéressée. Sélective, car ils réservent leur appui à ceux qui, à leurs yeux, le méritent, c'est-à-dire aux " bons chrétiens " provisoirement endettés. " Tandis que les mendiants, voilà la peste, le mal absolu dont il faut se débarrasser ${ }^{37}$. " Le pauvre qui refuse le modèle de vie chrétienne promu par les congréganistes n'a plus sa place dans la société : sa place est à l'hôpital général. Intéressée, car ce n'est pas une soif de justice sociale qui anime les confrères mais bien plutôt le désir de n'être point damnés. Ce n'est pas tant l'individu secouru qui importe que la vertu sanctifiante de l'aumône accomplie. On mesure donc ici la difficulté qui entoure toute entreprise en vue de cerner au plus près la véritable nature du sentiment religieux qui animait les congrégations mariales.

\section{La spiritualité des congréganistes de Saint-Thomas}

Quel chrétien était donc le congréganiste de Saint-Thomas? Était-il plus proche du Tartuffe de Molière ou des adeptes de Saint François de Sales? Pour tenter de répondre, il faut prendre en compte les manifestations les plus diverses de la foi des confrères. Un goût indéniable pour le faste transparaît d'abord dans la manière de solenniser les fêtes du calendrier liturgique. On consomme beaucoup de cierges et d'encens. Chez les Messieurs, on fait régulièrement appel à des choristes et parfois à des musiciens professionnels $^{38}$. Chez les marchands, la musique tient une place encore plus importante : les registres mentionnent en effet à partir de 1738 l'existence de " chantres ", c'est-à-dire d'environ cinq confrères chargés d'apprendre les chants aux nouveaux venus et de les entonner au bon moment au cours des offices ${ }^{39}$. La théâtralité n'est pas absente non plus

37. Chatellier, Louis, L'Europe des dévots, p. 149-150.

38. Ainsi, en 1706, ont-ils recours à l'organiste de la cathédrale Colesse. C'est la dernière fois qu'est utilisé l'orgue de la congrégation des messieurs puisqu'il est vendu l'année suivante aux Augustins.

39. Cet élément typique des grandes congrégations semblerait confirmer l'hypothèse avancée plus haut d'effectifs importants chez les marchands. 
des pratiques congréganistes. La prédication, qui à cette époque s'apparente parfois à un spectacle mondain, en est un bon exemple ${ }^{40}$. Les messieurs louent chaque année des chaises aux meilleures places dans la chapelle du collège (ils sont même assis dans le chœur en 1738!). C'est ainsi qu'ils ont assisté aux débuts d'un des plus grands prédicateurs du XVII siècle, le père Bourdaloue, qui passe au collège de Rennes en 1667-1668 ${ }^{41}$. Les processions, enfin, participent à leur manière de cette mise en scène du sacré. Les marchands et artisans défilent par exemple dans les rues de Rennes à la Conception, à l'Épiphanie, à la Purification, aux trois jours de Carnaval, à l'Annonciation, à l'Ascension, à l'octave du Saint-Sacrement, à l'Assomption et à la Nativité de la Vierge. À l'évidence, il n'y a pas pour eux contradiction entre dévotion et ostentation. Tout au contraire, les sodalités jésuites, à l'instar des anciennes fraternités, "mettaient leur dévotion dans la dimension et le poids des cierges bénis comme dans l'éclat de leurs croix et la richesse de leurs bannières ${ }^{42}$ ". Et quand leurs processions ne prennent plus symboliquement possession de l'espace urbain ${ }^{43}$, c'est la ville qui vient à leurs devants lors de leurs fêtes respectives. À cette occasion, les congrégations se rapprochent de leurs ancêtres les confréries : les marchands font ainsi provision de fleurs, blanchissent les nappes, empruntent des tableaux et des tapisseries à d'autres paroisses et, le jour venu, chargent un "réveilleur ", sorte de crieur, de " publier la feste " que des affiches ont déjà annoncée, tandis qu'un canonnier fait parler la poudre comme il est d'usage en ce temps pour tout événement joyeux. Les officiers de la seconde congrégation tirent même prétexte de la fête pour faire bombance! Néanmoins, réduire la spiritualité des assemblées de SaintThomas à ces manifestations ponctuelles serait méconnaître l'esprit baroque qui est esprit de contraste. L'éclat du faste n'avait de sens que s'il était le revers d'un quotidien plus austère et plus secret.

L'existence d'une " Aa " ou " Assemblée d'amis " à Saint-Thomas ne fait plus aucun doute ${ }^{44}$. Cette association secrète de piété, fréquente dans les collèges de la Compagnie de Jésus, se recrutait exclusivement parmi l'élite de la congrégation mariale et avait pour but d'animer secrètement celle-ci. Celle de Saint-Thomas, réunissant des élèves de philosophie, de théologie à des officiers de la congrégation des messieurs, imprima notamment sa

40. Deregnaucourt, Gilles et Poton, Didier, La vie religieuse en France aux XVI ${ }^{e}-X V I I^{e}-X V I I{ }^{e}$ siècles, Paris, 1995.

41. Lauras, M., Bourdaloue, sa vie et ses œuvres, Paris, 1881, t. 1. Il devait sans nul doute y avoir bon nombre de confrères dans l'assistance lorsque Bourdaloue prononça un panégyrique de saint François de Sales le 16 juin 1668 au couvent de la Visitation.

42. Villemarque, Théodore Hersart de, "Les fraternités bretonnes ", Association bretonne, 1880, [rééd. Libr. Académique Perrin, 2001].

43. VenARD, Marc, " Les confréries dans l'espace urbain : l'exemple de Rouen ", Annales de Bretagne et des Pays de l'Ouest, t. 90, 1983.

44. Michel, J., Aux origines de la Congrégation du Saint-Esprit : l'influence de l'Aa, association de piété, sur Claude-François Poullart des Places, Paris, 1992 : " Il est certain qu'il y eut à Rennes une Aa de théologiens; on ne connaît ni la date de sa fondation ni celle de sa disparition; on sait seulement qu'elle fut relevée par celle de Paris en 1742." 
marque sur la spiritualité de Claude-François Poullart des Places et de Louis Grignion de Montfort ${ }^{45}$. L'autre société secrète directement liée aux messieurs était la Compagnie du Saint-Sacrement. Cette dernière, on le sait, est souvent présentée par les spécialistes comme une " extension " des congrégations jésuites ${ }^{46}$. De fait, le lien entre la filiale rennaise de la Compagnie du Saint-Sacrement créée en 1649 et les sodalités jésuites de Saint-Thomas, longtemps supputé sans être pour autant prouvé, est désormais établi ${ }^{47}$.

Si l'on exclut ce qu'ont de trop particulier le faste et le secret, la question de savoir ce qui distingue au quotidien la spiritualité des congrégations mariales de celle des confréries traditionnelles reste entière. Notons d'abord que les enterrements de laïcs n'étaient pas autorisés dans la chapelle du collège et dans les deux chapelles des congrégations car elles étaient toutes bâties sur des terrains appartenant à la ville ${ }^{48}$. Mais ajoutons aussitôt que les sodalités compensaient aisément ce handicap grâce aux indulgences qu'elles délivraient en maintes occasions et surtout grâce à une forme de piété particulièrement exigeante. Cette dernière apparaît discriminante si l'on se souvient que les exercices de piété sont individuels et mensuels dans les confréries paroissiales mais collectifs et hebdomadaires dans les congrégations jésuites ${ }^{49}$. En général, les congréganistes s'assemblent le samedi vers 17 heures pour écouter les exhortations du père directeur et réciter à voix haute les litanies à la Vierge, et le dimanche à 7 heures pour suivre l'office des matines puis la messe et les laudes. Cet

45. D’après J. Michel, " la participation de Claude Poullart à une Aa rennaise d'humanistes et de philosophes serait la meilleure explication de sa cooptation, anormalement rapide, par celle de Louis-le-Grand en 1701. " "Quant à Louis Grignion, sa philosophie terminée, il commença sa théologie à Rennes qu'il ne quittera pour Paris qu'à la fin de 1692 ou au début de 1693. Il eut donc le temps d'être reçu dans l'Assemblée des théologiens et de recevoir le manuel de l'Aa, Pratique des Vertus. [...] C'est dans ce livre secret qu'il puisera des points de sa dévotion mariale dont, jusqu'à ce jour, les spécialistes de sa spiritualité ont vainement cherché la source. "

46. Fouqueray, R. P., Histoire de la Compagnie de Jésus en France des origines à la suppression (1528-1762), Paris, 1910-1913, t. V, p. 274. Voir aussi ScHIMBERG, A., L'Éducation morale dans les collèges de la Compagnie de Jésus sous l'Ancien Régime, Paris, 1913.

47. Voir Pocquet, B., La Compagnie du Saint-Sacrement à Rennes, Paris, 1904 et Rebelliau, A., La Compagnie secrète du Saint-Sacrement (1639-1662), Paris, 1908. La liste fournie par A. Rebelliau pour la filiale rennaise donne le nom du prêtre Fourel de La Bagotaie qui fut également secrétaire de la congrégation des messieurs en 1657 et membre du conseil jusqu'à sa mort en 1659. Il est difficilement envisageable que ce cas soit unique dans la mesure où ce prêtre figurait parmi les instances dirigeantes de la congrégation.

48. À titre de comparaison, chez les Carmes les plus fortunés des confrères pouvaient se faire enterrer dans le cloître. La grande famille des Robien, par exemple, y avait ses enfeus. Cf. PoulpiQuet, Dominique de, art. cit. supra.

49. JAMET, Catherine, "Les confréries de dévotion dans le diocèse de Rennes ", Annales de Bretagne et des Pays de l'Ouest, t. 87, 1980, p. 484 : " L'aspect collectif et fraternel, que les confréries se plaisent à exalter, est somme toute secondaire dans la pratique religieuse des confrères. Il est bien recommandé d'assister le plus souvent possible aux services religieux, mais la présence obligatoire n'est requise que pour un nombre réduit d'entre eux, tout le reste est affaire de piété individuelle. " 
encadrement communautaire de la piété a conduit parfois les congréganistes à prendre « l'habitude [...] d'entendre la messe, dimanche et jours de fête, en l'église des jésuites, voire en leur propre chapelle ${ }^{50}$ ". Qu'en étaitil à Saint-Thomas? Le récit de l'Établissement de la Congrégation des Marchands et Artisans précise bien que les horaires de la récitation du chapelet ont été choisis de sorte que " les Confrères sortant un peu devant huit heures en esté et devant neuf en hyver ayent une heure franche entre les Exercices de la Congrégation et la Messe Paroissiale à Laquelle tous conclurent d'assister le Dimanche autant que fere se pourroit pour l'Exemple public et montrer qu'un bon Congréganiste est un bon Paroissien ${ }^{51}$ ". De même chez les messieurs le règlement stipule que " tous les exercices se feront de manière que l'on puisse commodément se rendre à l'Office public des Paroissiens, comme tous les Congréganistes sont particulièrement exhortez à le faire ${ }^{52}$ ". Remarquons pour terminer que les membres des assemblées jésuites se doivent d'être exemplaires en dehors de la sodalité mais aussi et surtout en dedans. Obligation leur est faite d'" entretenir une parfaite union [...] qui soit une image de celle qu'on vit autrefois régner entre les premiers Fidelles ". Ce n'est pas en vain qu'ils s'appellent entre eux " confrères ». Les nombreux sobriquets et les multiples marques d'affection qu'ils se portent sont là pour l'attester. Plus profondément encore, ce vocatif traduit la volonté de réaliser au sein de la sodalité l'égalité des êtres devant Dieu : on ne juge plus son prochain d'après son rang ou sa richesse mais sur l'authenticité de sa ferveur. En 1709, les messieurs abandonnent volontairement l'étiquette : lorsque le Président au Parlement Lezonnet, alors préfet, demanda que fût fait un nouveau catalogue des confrères, "il fust arresté [...] que les noms y seroient mis selon l'ordre alphabétique sans autre distinction, ceux qui auroient eu plus de droit d'en demander l'ayant souhaité ${ }^{53}$ ". Ce type de décision tendait, bien sûr, à gommer les différences d'état entre clercs et laïcs et, partant, à rendre plus flous leurs rôles respectifs. D'ailleurs, comme le fait remarquer A. Châtellier, l'implication sans cesse plus forte des laïcs dans le déroulement des offices - en l'occurrence, à Saint-Thomas, surtout à partir de l'élection du premier préfet laïc en 1681 - a dû donner à certains un

50. CHATELLIER, Louis, op. cit., p. 154.

51. Arch. dép. d'Ille-et-Vilaine, 3D-15 et 16, Registre des élections.

52. Bibliothèque municipale de Rennes, 76. 756, Des Congrégations de Notre-Dame, Rennes, 1705, p. 48. Sur ce sujet les congrégations rennaises se conformaient à la règle générale rappelée par le R. P. Cerutti dans son Apologie de l'Institut et de la doctrine des Jésuites, Avignon, 1763, p. 246-249 : « De quoi accuse-t-on encore les congrégations? [...] D'entasser des Paroisses sur des Paroisses et de dispenser par des Bulles d'assister aux Offices des Eglises. Imputation odieuse et maligne puisqu'on n'y a jamais fait mention d'aucune Bulle qui dispensât les Fidèles d'assister aux Offices de leurs Paroisses, et qu'on tient au contraire ces assemblées à des heures différentes afin qu'on puisse accomplir les œuvres de surérogation sans manquer celles d'obligation. " Alphonse Poirier, dans Les Jésuites au tribunal de La Chalotais, Nantes, 1879, ajoute qu' en plusieurs endroits, c'est l'évêque lui-même qui a fixé ces heures ".

53. Arch. dép. d'Ille-et-Vilaine, 3D-14, Annales. 
comportement quasi ecclésiastique ${ }^{54}$. Cette religion communautaire où toute prière, même la plus intérieure, se fait à haute voix et dans laquelle les laïcs empiètent sur les tâches dévolues aux prêtres fournira des armes à la critique janséniste, à commencer par les Provinciales de Pascal ${ }^{55}$. Essayer d'atteindre la perfection tout en accomplissant son devoir d'état, tel était l'objectif, apparemment simple, de l'« humanisme dévot ${ }^{56}$ ". C'était en fait, on l'a vu, une piété qui n'excluait ni le faste ni le goût du secret et pouvait donc osciller entre une vaine ostentation sociale et la simple bigoterie, tout en passant par de véritables élans mystiques ${ }^{57}$.

La suppression de la Compagnie de Jésus en 1762 mit fin brutalement aux pieuses assemblées de Saint-Thomas. À Rennes, les deux congrégations mariales furent les premières victimes de la virulence du procureur général Caradeuc de La Chalotais ${ }^{58}$. À vrai dire, on ne s'explique pas comment elles ont pu aussi facilement se laisser réduire à néant. Leur fin apparaît a posteriori d'autant plus énigmatique que leur influence sur l'élite locale, et tout particulièrement sur le milieu parlementaire, était réelle. Mais le plus surprenant est qu'il ne soit pratiquement rien resté d'elles, hormis un certain " esprit marial " que d'aucuns revendiquèrent par la suite. Aujourd'hui, seul le tableau du maître-autel de l'église Toussaints, intitulé La Vierge protégeant les congréganistes, témoigne encore de cette part du passé oubliée de la ville de Rennes et de ses habitants.

54. ChATELliER, Louis., op. cit., p. 183 : " Allure modeste et digne dans la physionomie, une onction dans la voix, un extérieur austère et dépourvu d'élégance : tout cela fait qu'en observant un dévot sortir de l'oratoire et marcher modestement, les yeux baissés, enveloppé dans son manteau noir, un passant peut à bon droit se demander s'il ne s'agit pas d'un homme d'Église. "

55 . Notamment la neuvième provinciale.

56. Cf. Lebrun, François (dir.), Histoire de la France religieuse, t. 2, p. 348 et suivantes.

57. Dans Les Jésuites et le secret de leur puissance, Paris, 1933, R. Fülöp-Miller rappelle que la mystique inaugurée par Ignace de Loyola allait à rebours de la mystique traditionnelle. En refusant le détachement de toute chose qui, selon lui, conduisait à une déshumanisation de la personne, mais, au contraire, en réhabilitant les sens pour marcher sur les traces de la sainteté, en refusant de considérer la rencontre avec Dieu comme une grâce, mais, au contraire, en en faisant le fruit d'une ascèse rationnelle et volontaire, le père fondateur définissait une nouvelle pratique de la foi promise à un long avenir.

58. Tandis que la dissolution de la Compagnie de Jésus dans le ressort du Parlement de Bretagne est prononcée le 23 décembre 1761, La Chalotais souhaite dès la lecture de ses Comptes rendus, début décembre, que soient interdites les congrégations mariales de Saint-Thomas. 
RESUME

Version urbaine et élitiste des célèbres " missions ", les " congrégations " dédiées à la Vierge ont véritablement incarné le militantisme tridentin des Jésuites sous l'Ancien Régime. Le collège Saint-Thomas de Rennes fournit un bon exemple de cette stratégie apostolique, lui qui abrita deux congrégations où se retrouvaient tant les nobles du Parlement que les membres de la bourgeoisie. Plus structurées, plus riches et vraisemblablement plus pieuses que les confréries traditionnelles, elles ont marqué la ville de leur empreinte spirituelle.

\section{ABSTRACT}

The "congregations" dedicated to the Virgin, urban and elitist versions of the famous "missions", traly embodied the Jesuits' tridentine militancy at the time of the Ancien Régime. The "College Saint-Thomas de Rennes", which welcomed two congregations where were found noblemen from the Parliament as well as members of the bourgeoisie, provides a good example of this apostolic strategy. More structured, richer and, in all likelihood, more devout than the traditional brotherhoods, those congregations have put their spiritual stamp on the town. 